\title{
Dipeptidyl Peptidase-4 Inhibitors Reduced Long- term Cardiovascular Risk in Japanese Diabetic Patients After Percutaneous Coronary Intervention via Insulin-like Growth Factor-1 Axis: A 10-year Follow-up Study
}

\section{Yuichi Chikata}

Juntendo University School of Medicine Graduate School of Medicine: Juntendo Daigaku Igakubu Daigakuin Igaku Kenkyuka

Hiroshi Iwata ( $\nabla$ hiroiwata-circ@umin.ac.jp )

Juntendo University School of Medicine https://orcid.org/0000-0001-7098-9406

\section{Katsutoshi Miyosawa}

Juntendo University School of Medicine Graduate School of Medicine: Juntendo Daigaku Igakubu Daigakuin Igaku Kenkyuka

\section{Takuma Koike}

Juntendo University School of Medicine Graduate School of Medicine: Juntendo Daigaku Igakubu Daigakuin Igaku Kenkyuka

\section{Hidetoshi Yasuda}

Juntendo University School of Medicine Graduate School of Medicine: Juntendo Daigaku Igakubu Daigakuin Igaku Kenkyuka

\section{Takehiro Funamizu}

Juntendo University School of Medicine Graduate School of Medicine: Juntendo Daigaku Igakubu Daigakuin Igaku Kenkyuka

\section{Shinichiro Doi}

Juntendo University School of Medicine Graduate School of Medicine: Juntendo Daigaku Igakubu Daigakuin Igaku Kenkyuka

\section{Hirohisa Endo}

Juntendo University School of Medicine Graduate School of Medicine: Juntendo Daigaku Igakubu Daigakuin Igaku Kenkyuka

\section{Hideki Wada}

Juntendo Shizuoka Hospital: Juntendo Daigaku Igakubu Fuzoku Shizuoka Byoin

\section{Ryo Naito}

Juntendo University School of Medicine Graduate School of Medicine: Juntendo Daigaku Igakubu Daigakuin Igaku Kenkyuka 


\section{Manabu Ogita}

Juntendo Shizuoka Hospital: Juntendo Daigaku Igakubu Fuzoku Shizuoka Byoin

\section{Tomotaka Dohi}

Juntendo University School of Medicine Graduate School of Medicine: Juntendo Daigaku Igakubu Daigakuin Igaku Kenkyuka

\section{Takatoshi Kasai}

Juntendo University School of Medicine Graduate School of Medicine: Juntendo Daigaku Igakubu

Daigakuin Igaku Kenkyuka

\section{Kikuo Isoda}

Juntendo University Nerima Hospital: Juntendo Daigaku Igakubu Fuzoku Nerima Byoin

\section{Shinya Okazaki}

Juntendo University School of Medicine Graduate School of Medicine: Juntendo Daigaku Igakubu

Daigakuin Igaku Kenkyuka

\section{Katsumi Miyauchi}

Juntendo Tokyo Koto Geriatric Medical Center: Juntendo Tokyo Koto Koreisha Iryo Center

\section{Tohru Minamino}

Juntendo University School of Medicine Graduate School of Medicine: Juntendo Daigaku Igakubu Daigakuin Igaku Kenkyuka

\section{Original investigation}

Keywords: Dipeptidyl peptidase-4 inhibitors, percutaneous coronary intervention, diabetes mellitus, insulin-like growth factor-1, insulin-like growth factor-binding protein 3

Posted Date: July 26th, 2021

DOI: https://doi.org/10.21203/rs.3.rs-730348/v1

License: (a) (1) This work is licensed under a Creative Commons Attribution 4.0 International License. Read Full License 


\section{Abstract}

Background: Dipeptidyl-peptidase-4 inhibitors (DPP4i) have become one of the most used antidiabetic medications worldwide due to their good safety profiles and tolerability with a low risk of hypoglycemia, however, no large cardiovascular outcome trials (CVOTs) have shown any significant superiority with respect to reducing cardiovascular risk. On the contrary, since observational studies have suggested the effects of DPP4i are enhanced some populations, such as Asians and those who without overweight, their prognostic benefit is still under debate. Therefore, the aim of this study was to assess the prognostic impact of DPP4i in patients with both diabetes and coronary artery disease (CAD) through the insulin-like growth factor-1 (IGF-1) axis, a substrate of DPP4 and it is relevant for the cardiovascular system.

Methods: This single-center analysis involved consecutive Japanese diabetic patients who underwent percutaneous coronary intervention (PCl) for the first time between 2008 and $2018(n=885)$. Primary and secondary endpoints were set as cardiovascular (CV)-death and the composite of CV-death, non-fatal myocardial infarction and ischemic stroke (3P-MACE). Serum levels of IGF-1 and its main binding protein (IGF- binding protein 3: IGFBP-3) were measured by ELISA.

Results: Unadjusted Kaplan-Meier analyses revealed reduced incidences of CV-death and 3P-MACE by DPP4i, which was particularly enhanced in patients who were not overweight (BMI $\leq 25)$. Multivariate Cox hazard analyses consistently indicated reduced risks of CV-death by DPP4i at PCl (hazard ratio (HR): $0.39,95 \%$ confidence interval $(\mathrm{Cl}): 0.16-0.82, \mathrm{p}=0.01$ ) and 3P-MACE (HR: $0.47,95 \% \mathrm{Cl}: 0.25-0.84$, $\mathrm{p}=0.01$ ), respectively. Moreover, elevated IGF-1 activity indicated by the IGF-1/IGFBP-3 ratio was associated with decreased risks of both endpoints and it was significantly higher in patients with DPP4i $(p<0.0001)$.

Conclusion: The findings of the present study indicate that DPP4i improved outcomes in Japanese diabetic patients following PCl, in which the beneficial effects might be mediated by DPP4-IGF-1 axis.

Trial registration: This study reports the retrospective analysis of a prospective registry database of patients who underwent PCl at Juntendo University Hospital, Tokyo, Japan (Juntendo Physicians' Alliance for Clinical Trials, J-PACT), which is publicly registered (University Medical Information Network Japan - Clinical Trials Registry, UMIN-CTR 000035587).

\section{Background}

Diabetes mellitus, an established risk for atherosclerotic cardiovascular (CV) mortality and morbidity, such as coronary, cerebral and peripheral artery disease, is a major socioeconomic burden worldwide [1, 2]. Appropriate glycemic control in combination with multifactorial risk control is thus essential in order to avoid macrovascular complications that can lead to critical consequences in diabetic patients. Among multiple lines of anti-diabetic medications, dipeptidyl peptidase-4 inhibitors (DPP4i) are a major antidiabetic agent class in the United States [3], European Union [4, 5] and Japan [6]. Although accumulated experimental evidence has suggested an atheroprotective effects of DPP4 inhibition [7-9], 
previous major large-scale cardiovascular outcome trials (CVOTs) of DPP4i which primarily aimed to establish their non-inferiority compared to conventional anti-diabetic therapy, have not demonstrated a clear benefit with respect to improving outcomes in populations which mainly consisted of diabetic individuals with a history of CV disease (secondary prevention) [10-13]. In contrast, 2 observational studies have reported differences in the efficacy of DPP4i among populations, including the superiority of the glucose lowering effect of DPP4i in Asians and normal weight compared to obese individuals [14, 15]. Moreover, a study using a Korean national database showed the substantial prognostic merit of DPP4i at reducing cardiovascular risk, and the composite of all-cause death, stroke and myocardial infarction in a population without a history of cardiovascular disease [16]. Accordingly, the differences regarding the prognostic impact of DPP4i among subgroups remain obscure and inconclusive.

In this study, we thus investigated the prognostic efficacy of DPP4i in Japanese patients with diabetes following percutaneous coronary intervention $(\mathrm{PCl})$ by using a prospective single-center $\mathrm{PCl}$ registry database. Moreover, to elucidate the potential target molecules of DPP4 and the benefits of its inhibition, we measured the serum levels of insulin-like growth factor-1 (IGF-1), as one of the substrates for catalytic degradation by DPP4 and its regulatory protein, insulin-like growth factor-binding protein 3 (IGFBP-3), and assessed their prognostic effects in association with the use of DPP4i.

\section{Patients And Methods}

This study followed the Declaration of Helsinki and was approved by the Institutional Review Board (IRB) of Juntendo University (IRB-ID: 20-287). The single-center prospective registry database of patients who underwent any type of $\mathrm{PCl}$ at Juntendo University Hospital, Tokyo, Japan (Juntendo Physicians' Alliance for Clinical Trial, J-PACT) since 1984 is publicly registered (University Medical Information Network Japan - Clinical Trials Registry, UMIN-CTR 000035587). Written informed consent was obtained from all participants for the J-PACT registry.

\section{Participants, endpoints, follow-up, and follow-up duration}

This study is a retrospective analysis of a portion of the J-PACT registry database involving consecutive 885 diabetic patients out of 4039 entire patients who underwent any type of $\mathrm{PCI}$ for coronary artery disease (CAD) for the first time at Juntendo University Hospital, Tokyo, Japan between December 2008 and January 2018. Diabetes was defined as $\mathrm{HbA} 1 \mathrm{c} \geq 6.5 \%$ or with any diabetic medications at $\mathrm{PCl}$ procedure in this study. Participants were divided into two groups; with or without dipeptidylpeptidase-4 inhibitors (DPP4i) (DPP4i (+) n=324, DPP4i (-) n=561) at PCl procedure, and the incidence and risk of subsequent endpoints following PCl were assessed (Supplementary Figure 1).

The primary endpoint was set as cardiovascular (CV)-related death and the secondary endpoint was the composite of cardiovascular death, non-fatal myocardial infarction and ischemic stroke (3-point major adverse cardiovascular events: 3P-MACE). In this prospective PCI registry database, patient follow-up was based on chart review, as far as they were followed at Juntendo University Hospital. A prognosis survey questionnaire was mailed out every 5 years if they were transferred to other institutions. When there was 
no response to the questionnaire, further follow-up was conducted by phone call. In cases in which no response was achieved by either, follow-up was terminated at the latest time point, at which their survival at our institution was confirmed, such as the last visit date to an outpatient clinic or the last day of any hospitalization. The median and the range of the follow up period were 1456 and 0-3785 days, respectively.

\section{Blood sampling and the measurement of serum levels of IGF-1 and IGFBP-3}

Blood samples were obtained immediately prior to $\mathrm{PCl}$ through an inserted blood access ( 5 to 8 French catheter) and stored at $-80^{\circ} \mathrm{C}$ until measurement of IGF-1 and IGFBP-3 concentrations $(n=816)$. Serum levels of IGF-1 and IGFBP-3 were determined using commercially available enzyme-linked immunosorbent assay (ELISA) kits (Duoset DY291 and DY675; R\&D Systems, USA) according to the manufacturer's protocol.

\section{Statistical analysis}

Quantitative variables are presented as the mean \pm standard deviation or median with interquartile range (IQR) in accordance with the results of the Shapiro-Wilk normality test. Categorical variables are presented as the numbers and percentages. Quantitative data between groups were compared using the Student's $t$-test or the Wilcoxon rank sum test. Unadjusted Kaplan-Meier analysis was used to evaluate the time to the cumulative incidence of endpoints followed by the log-rank test for comparisons. Univariate and multivariate analyses using the following models of Cox proportional hazards regression analyses (Model 1-4) were used to calculate the hazard ratios (HRs) of DPP4i and the IGF-1/IGFBP-3 ratio for endpoints. Variables used in Models 1-3 were selected based on the background demographics different between the groups and were associated with endpoints in univariate analyses. Model 1 included age (a continuous variable) and sex. Model 2 included age (a continuous variable), sex, body mass index (BMI) (a continuous variable), chronic kidney disease ( $\geq$ stage 3 ), b-type natriuretic peptide (BNP) (a continuous variable) and insulin. Model 3 included age (a continuous variable), sex, acute coronary syndrome (ACS), left ventricular ejection fraction (LVEF), statins, and hemoglobin. Additionally, Model 4 included other diabetic medications, including metformin, sulfonylurea, alpha-glucosidase inhibitors, thiazolidinedione, glinide and insulin. Statistical significance was defined as a p-value $<0.05$ and analyses were performed using statistical software (JMP Pro 12.0; SAS Institute Inc., Cary, NC, USA and IBM SPSS Statistics, Version 26.0. Armonk, NY, USA).

\section{Results}

\section{Baseline characteristics in patients with or without DPP4i}

The background demographics, comorbidities and medications are listed and compared between groups with and without DPP4i in Table 1. Most of the background demographic parameters, including age, sex, $\mathrm{BMI}$, and diabetes duration were similar, while the proportion of ACS was significantly higher in the DPP4i $(-)$ group. Parameters regarding target lesions and number of diseased vessels were not different 
between the two groups. Total cholesterol, low-density lipoprotein-cholesterol (LDL-C) and glycated hemoglobin ( $\mathrm{HbA1c-NG}$ ) levels were lower in patients with DPP4i compared to those without. With respect to conventional antidiabetic medications, the prevalence of insulin use was almost doubled in the DPP4i (-) group compared to the DPP4(+) group, while that of metformin was higher in the DPP4i (+) group. Despite a low prevalence, the use of sodium-glucose cotransporter 2 (SGLT2) inhibitors was higher in the DPP4i (+) group. The ratios of statins and ezetimibe use were higher in the DPP4i (+) group in concert with lower LDL-C.

\section{Prognostic merit of DPP4i in patients after PCI}

During the follow-up period after PCl, the numbers of identified CV deaths and 3P-MACE were 59 (6.7\%) and $89(10.1 \%)$ out of 885 participants, respectively. Unadjusted Kaplan-Meier analysis followed by the log-rank comparison test showed that the cumulative incidences of both primary and secondary endpoints were significantly lower in the DPP4i (+) group compared to the DPP4i (-) group indicating a possible favorable prognostic effect of DPP4i in this population following PCl (Figure 1a). Of interest, such an impact of DPP4i was significant only in individuals who were not overweight $(B M I \leq 25)$, while the cumulative incidences of both CV death and 3P-MACE in the DPP4i (+) and (-) groups were similar amongst overweight patients and obese patients (Figure 1b).

Univariate Cox proportional hazard analyses for calculating hazard ratios for subsequent CV death and 3P-MACE after PCI revealed that DPP4i at procedure was significantly associated with reduced risk of both endpoints (Supplementary Table 1 and 2). Multivariate Cox proportional analyses using 3 models (Models 1-3) in which covariates were selected based on background demographics and univariate analyses (Supplementary Table 1 and 2) showed that DPP4i at procedure significantly lowered the risks of CV death and 3P-MACE (Figure 3). Furthermore, multivariate analyses using another model (Model 4), which included diabetic medications, such as metformin, sulfonylurea, alpha-glucosidase inhibitors, thiazolidinedione, glinide, and insulin in addition to DPP4i indicated that only DPP4i was associated with lower risk of both endpoints (Figure 3).

\section{Beneficial effect of DPP4i exerted potentially through IGF-1 axis}

In an attempt to elucidate a portion of the potential mechanisms which mediate the favorable prognostic impact of DPP4i in the present population, we measured the serum concentrations of IGF-1, one of the major substrates of DPP4 [17], and its regulatory molecule IGFBP-3 in the entire population. As shown in Figure 2, despite no significant difference in the serum levels of IGF-1 in the two groups $(76.3 \mathrm{ng} / \mathrm{mL}$ $[57.3,104.3]$ vs. $73.9 \mathrm{ng} / \mathrm{mL}[54.7,98.2], \mathrm{p}=0.30)$, IGFBP-3 levels were significantly attenuated $(519.3 \pm$ $218.1 \mathrm{ng} / \mathrm{mL}$ vs. $650.8 \pm 241.7 \mathrm{ng} / \mathrm{mL}, \mathrm{p}<0.0001)$ and the ratio of IGF-1/IGFBP-3, which is a known indicator of IGF-1 activity [18], was significantly amplified in the DPP4i (+) group compared to the DPP4i $(-)$ group $(0.16[0.10,0.25]$ vs. $0.12[0.08,0.18], p<0.0001)$. Moreover, unadjusted Kaplan-Meier analyses when dividing the participants into two groups according to the median of IGF-1/IGFBP-3 revealed that the cumulative incidences of both CV death and 3P-MACE were constantly and significantly lower in patients with a high IGF-1/IGFBP-3 ratio (Supplementary Figure 2a). Furthermore, differences in the 
cumulative incidences of both endpoints between the high and low IGF-1/IGFBP-3 groups were enhanced in patients who were not overweight $(\mathrm{BMI} \leq 25)$ and attenuated in those who were overweight or obese (BMI > 25) (Supplementary Figure 2b), findings that were substantially comparable with those in KaplanMeier analyses when the participants were divided based on the presence or absence of DPP4i (Figure 1a and 1b). Moreover, multivariate analysis including age, sex, BMI, chronic kidney disease, BNP and insulin use (Model 2) revealed that an elevated IGF-1/IGFBP-3 ratio was associated with a significantly decreased risk for CV-death and 3P-MACE (Supplementary Table 4).

\section{Discussion}

This single-center observational study of a prospective $\mathrm{PCl}$ registry database involving consecutive Japanese diabetic patients primarily demonstrated that the use of DPP4i at the time of PCl procedure was associated with avoiding subsequent CV death and 3P-MACE. The merit of DPP4i was considerably augmented in patients with a lower BMI. Moreover, the ratio of IGF-1 to IGFBP-3, one of the enzymatic substrates of DPP4 to its main transport protein indicating the bioavailability of IGF-1, was significantly higher in patients with DPP4i. Kaplan-Meier analyses, as well as unadjusted and adjusted Cox proportional hazard analyses showed that a high IGF-1/IGFBP-3 ratio was associated with a significantly lower risk of CV death and 3P-MACE after PCI. Moreover, very similar to analyses with and without DPP4i, the prognostic impact of the IGF-1/IGFBP-3 ratio was enhanced in patients with a lower BMI.

DPP4, a multifunctional circulating or cell surface protein, is present on a wide range of cell types and exerts a variety of biological activities, such as protease activity, interaction with the extracellular matrix, and regulation of intracellular signaling $[17,19]$. Inhibition of DPP4 lowers blood glucose by inhibiting the catalytic degradation of the incretin hormones glucagon-like peptide-1 (GLP-1) and glucose-dependent insulinotropic polypeptide (GIP), both of which promote insulin secretion from pancreatic beta-cells, and inhibit glucagon secretion and glucose production in the liver $[20,21]$. Other than incretins, there are physiological substrates of DPP4 that are bioactive molecules cleaved by DPP4, such as BNP, neuropeptide Y, stromal cell-derived factor-1 a (SDF-1a) and IGF-1 [17, 22]. Multiple lines of previous experimental evidence have demonstrated the cardioprotective and anti-atherogenic effects of DPP4i [79]. However, landmark large-scale randomized CVOTs of DPP4i which mainly involved patients with a history of CV disease (secondary prevention) did not show any clear benefit for reducing the risk of the composite of cardiovascular events [10-13]. Although these studies had been primarily designed to identify the non-inferiority, and not the superiority, of DPP4i compared to conventional diabetic medications, the study results and their clinical impacts were considerably different to those of SGLT-2 inhibitors $[23,24]$ and GLP-1 receptor agonists $[25,26]$. However, observational studies have indicated that the effects of DPP4i might not be uniform among ethnicities or those with different BMI $[14,15]$. The Diabetes Epidemiology: Collaborative analysis Of Diagnostic criteria in Europe (the DECODE) and in Asia (the DECODA) studies from the World Health Organization (WHO) demonstrated that both insulin resistance and secretion capacity are higher in Caucasians than Asians [27-29]. In Western countries, insulin resistance, which frequently occurs in conjunction with obesity, is the predominant pathophysiological issue, while impaired insulin secretion is a major issue in Asians [30] and a genetic 
difference in relation to diabetes between Asians and non-Asians has been also reported [31]. In addition to ethnicity, it has been reported BMI might alter the effects of DPP4i [14]. DPP4 enzymatic activity was suggested to be upregulated in proinflammatory situations, including obesity, and it was reported to be significantly positively correlated with BMI $[19,32]$.

Consistent with these previous findings regarding the possible superiority in the effects of DPP4i in Asians and individuals with low BMI, DPP4i in the present study was found to be beneficial at reducing the risk of $\mathrm{CV}$ death and 3P-MACE after PCI in the entire study population. Moreover, the merit of DPP4i was substantially enhanced in patients with a lower BMI $(\leq 25)$ and attenuated in those who were overweight $(\mathrm{BMI}>25)$. The present findings indicate that there might be subpopulations, such as Asians and those without overweight, who specifically benefit from DPP4i, although large scale CVOTs involving a limited number of Asians (9-20\%) [10-13] and patients with low BMI failed to show any significant merit.

Among substrates which are catalytically deactivated by DPP4, there is a large body of evidence suggesting the cardioprotective effects are mediated through the IGF-1 axis, which leads to the improvement of endothelial function, plaque stability, and the inhibition of vascular inflammation [3335]. It has been suggested that low circulating IGF-1 levels and high IGFBP-3 levels significantly increase the risk of developing ischemic heart disease in a primary prevention population [36]. In this study, serum IGF-1 levels were similar in both patients with and without DPP4i, but IGFBP-3 levels were significantly reduced and the IGF-1/IGFBP-3 ratio was significantly increased in patients with DPP4i. Since the IGF1/IGFBP-3 ratio has been considered to be an indicator of IGF-1 bioavailability [18], these findings suggest that administration of DPP4i might increase its bioavailability in this population, which in turn may lead to better outcomes. Consistently, prognostic analyses of the IGF-1/IGFBP-3 ratio by KaplanMeier and Cox proportional analyses have indicated its elevation was significantly associated with a reduced risk of adverse outcomes after PCI. Moreover, similar to that for DPP4i, the favorable prognostic impact of the IGF-1/IGFBP-3 ratio was substantially augmented in the population that was not overweight. Taking these findings together, the beneficial prognostic effects of DPP4i in this population may be exerted through the DPP4-IGF-1 axis.

\section{Limitations Of The Study}

There are several limitations to this study. First, it is single centered and retrospective and included only Japanese patients, and therefore did not compare findings amongst different ethnicities. The possibility that unknown confounders might have had an influence on the results could not be completely denied, even though multivariate analyses adjusted for baseline characteristics and known prognostic factors. Second, the number of patients treated with newer generations of antidiabetic medications, such as SGLT-2 inhibitors and GLP-1 receptor agonists, was quite small. This should have been taken into consideration as confounders of multivariate analyses. Third, this study did not assess heart failure hospitalization as an endpoint, although a trial revealed that a DPP4i, saxagliptin, was associated with a higher incidence of heart failure hospitalization compared to placebo [10]. Therefore, larger-scale and 
further updated future studies involving participants with newer classes of antidiabetic drugs should assess heart failure as an outcome as well.

\section{Conclusions}

This retrospective observational study demonstrated that patients treated with DPP4i and those with a higher IGF-1/IGFBP-3 ratio were associated with lower risks of CV mortality and 3P-MACE in Japanese diabetic patients after $\mathrm{PCl}$, especially in lower BMI patients. These findings suggest that the benefit of DPP4i might be mediated by the DPP4-IGF-1 axis.

\section{Abbreviations}

CV: cardiovascular; DPP4i: dipeptidyl-peptidase-4 inhibitors; CVOTs: cardiovascular outcome trials; PCl: percutaneous coronary intervention; IGF-1: insulin-like growth factor-1; IGFBP-3: insulin-like growth factorbinding protein 3; IRB: Institutional Review Board; CAD: coronary artery disease; 3P-MACE: 3-point major adverse cardiovascular events; IQR: interquartile range; HRs: hazard ratios; BMI: body mass index; BNP: btype natriuretic peptide; ACS: acute coronary syndrome; LVEF: left ventricular ejection fraction; LDL-C: lowdensity lipoprotein-cholesterol; HbA1 c-NG: glycated hemoglobin; SGLT-2: sodium-glucose cotransporter 2; GLP1: glucagon-like peptide-1; GIP: glucose-dependent insulinotropic polypeptide; SDF-1a: stromal cellderived factor-1a

\section{Declarations}

Acknowledgements: The authors wish to thank Ms. Yumi Nozawa and Ms. Emiko Nakamura for their significant contributions to this study.

Authors' contributions: Y.C.: Data Curation, Formal analysis, Writing-Original Draft. H.I.: Formal analysis, Writing-Review and Editing, Conceptualization, Methodology and Supervision. K.M.: Formal analysis, Supervision. T.K., H.Y.: Data Curation. T.F., S.D., H.E., H.W., R.N., M.O., T.D., T.K.: Data Curation, Project administration. K.I., S.O., K.M., T.M.: Writing-Review and Editing, Supervision. All authors read and approved the final manuscript.

Funding: This study was supported by a Grant-in-Aid for Scientific Research from the Ministry of Education, Culture, Sports, Science and Technology of Japan (18K08049).

Availability of data and materials: The datasets used and/or analyzed during the current study are available from the corresponding author on reasonable request.

Ethics approval and consent to participate: This study was performed in accordance with the Declaration of Helsinki and was approved by the Institutional Review Board (IRB) of Juntendo University (IRB-ID: 20287), and all patients gave their written informed consent to participate in the study. 
Consent for publication: Not applicable.

Competing interests: All authors have nothing to declare.

\section{References}

1. Tancredi M, Rosengren A, Svensson AM, Kosiborod M, Pivodic A, Gudbjörnsdottir S, Wedel H, Clements M, Dahlqvist S, Lind M. Excess Mortality among Persons with Type 2 Diabetes. $\mathrm{N}$ Engl J Med. 2015;373(18):1720-32.

2. Bommer C, Heesemann E, Sagalova V, Manne-Goehler J, Atun R, Bärnighausen T, Vollmer S. The global economic burden of diabetes in adults aged 20-79 years: a cost-of-illness study. Lancet Diabetes Endocrinol. 2017;5(6):423-30.

3. Raval AD, Vyas A. National Trends in Diabetes Medication Use in the United States: 2008 to 2015. J Pharm Pract. 2020;33(4):433-42.

4. Wilkinson S, Douglas I, Stirnadel-Farrant H, Fogarty D, Pokrajac A, Smeeth L, Tomlinson L. Changing use of antidiabetic drugs in the UK: trends in prescribing 2000-2017. BMJ Open. 2018;8(7):e022768.

5. Engler C, Leo M, Pfeifer B, Juchum M, Chen-Koenig D, Poelzl K, Schoenherr H, Vill D, Oberdanner J, Eisendle $\mathrm{E}$, et al: Long-term trends in the prescription of antidiabetic drugs: real-world evidence from the Diabetes Registry Tyrol 2012-2018. BMJ Open Diabetes Res Care 2020, 8(1).

6. Katakami N, Mita T, Takahara M, Yajima T, Wada F, Kawashima M, Shimomura I, Watada H. Baseline Characteristics of Patients with Type 2 Diabetes Initiating Second-Line Treatment in Japan: Findings from the J-DISCOVER Study. Diabetes Ther. 2020;11(7):1563-78.

7. Akita K, Isoda K, Shimada K, Daida H. Dipeptidyl-peptidase-4 inhibitor, alogliptin, attenuates arterial inflammation and neointimal formation after injury in low-density lipoprotein (LDL) receptor-deficient mice. J Am Heart Assoc. 2015;4(3):e001469.

8. Hirano T, Yamashita S, Takahashi M, Hashimoto H, Mori Y, Goto M. Anagliptin, a dipeptidyl peptidase-4 inhibitor, decreases macrophage infiltration and suppresses atherosclerosis in aortic and coronary arteries in cholesterol-fed rabbits. Metabolism. 2016;65(6):893-903.

9. Suda M, Shimizu I, Yoshida Y, Hayashi Y, Ikegami R, Katsuumi G, Wakasugi T, Yoshida Y, Okuda S, Soga T, et al. Inhibition of dipeptidyl peptidase-4 ameliorates cardiac ischemia and systolic dysfunction by up-regulating the FGF-2/EGR-1 pathway. PLoS One. 2017;12(8):e0182422.

10. Scirica BM, Bhatt DL, Braunwald E, Steg PG, Davidson J, Hirshberg B, Ohman P, Frederich R, Wiviott SD, Hoffman EB, et al. Saxagliptin and cardiovascular outcomes in patients with type 2 diabetes mellitus. N Engl J Med. 2013;369(14):1317-26.

11. White WB, Cannon CP, Heller SR, Nissen SE, Bergenstal RM, Bakris GL, Perez AT, Fleck PR, Mehta CR, Kupfer S, et al. Alogliptin after acute coronary syndrome in patients with type 2 diabetes. $\mathrm{N}$ Engl $\mathrm{J}$ Med. 2013;369(14):1327-35.

12. Green JB, Bethel MA, Armstrong PW, Buse JB, Engel SS, Garg J, Josse R, Kaufman KD, Koglin J, Korn $\mathrm{S}$, et al. Effect of Sitagliptin on Cardiovascular Outcomes in Type 2 Diabetes. N Engl J Med. 
2015;373(3):232-42.

13. Rosenstock J, Perkovic V, Johansen OE, Cooper ME, Kahn SE, Marx N, Alexander JH, Pencina M, Toto RD, Wanner C, et al. Effect of Linagliptin vs Placebo on Major Cardiovascular Events in Adults With Type 2 Diabetes and High Cardiovascular and Renal Risk: The CARMELINA Randomized Clinical Trial. Jama. 2019;321(1):69-79.

14. Kim YG, Hahn S, Oh TJ, Kwak SH, Park KS, Cho YM. Differences in the glucose-lowering efficacy of dipeptidyl peptidase-4 inhibitors between Asians and non-Asians: a systematic review and metaanalysis. Diabetologia. 2013;56(4):696-708.

15. Cai X, Han X, Luo Y, Ji L: Efficacy of dipeptidyl-peptidase-4 inhibitors and impact on $\beta$-cell function in Asian and Caucasian type 2 diabetes mellitus patients: A meta-analysis. J Diabetes 2015, 7(3):347359.

16. Ha KH, Kim B, Shin HS, Lee J, Choi H, Kim HC, Kim DJ. Comparative Cardiovascular Risks of Dipeptidyl Peptidase-4 Inhibitors: Analyses of Real-world Data in Korea. Korean Circ J. 2018;48(5):395-405.

17. Mulvihill EE, Drucker DJ. Pharmacology, physiology, and mechanisms of action of dipeptidyl peptidase-4 inhibitors. Endocr Rev. 2014;35(6):992-1019.

18. Ranke MB. Insulin-like growth factor binding-protein-3 (IGFBP-3). Best Pract Res Clin Endocrinol Metab. 2015;29(5):701-11.

19. Zhong J, Maiseyeu A, Davis SN, Rajagopalan S. DPP4 in cardiometabolic disease: recent insights from the laboratory and clinical trials of DPP4 inhibition. Circ Res. 2015;116(8):1491-504.

20. Gupta R, Walunj SS, Tokala RK, Parsa KV, Singh SK, Pal M. Emerging drug candidates of dipeptidyl peptidase IV (DPP IV) inhibitor class for the treatment of Type 2 Diabetes. Curr Drug Targets. 2009;10(1):71-87.

21. Drucker DJ. The Cardiovascular Biology of Glucagon-like Peptide-1. Cell Metab. 2016;24(1):15-30.

22. Avogaro A, Fadini GP. The pleiotropic cardiovascular effects of dipeptidyl peptidase-4 inhibitors. Br J Clin Pharmacol. 2018;84(8):1686-95.

23. Zinman B, Wanner C, Lachin JM, Fitchett D, Bluhmki E, Hantel S, Mattheus M, Devins T, Johansen OE, Woerle HJ, et al. Empagliflozin, Cardiovascular Outcomes, and Mortality in Type 2 Diabetes. N Engl J Med. 2015;373(22):2117-28.

24. Mahaffey KW, Neal B, Perkovic V, de Zeeuw D, Fulcher G, Erondu N, Shaw W, Fabbrini E, Sun T, Li Q, et al. Canagliflozin for Primary and Secondary Prevention of Cardiovascular Events: Results From the CANVAS Program (Canagliflozin Cardiovascular Assessment Study). Circulation. 2018;137(4):32334.

25. Marso SP, Daniels GH, Brown-Frandsen K, Kristensen P, Mann JF, Nauck MA, Nissen SE, Pocock S, Poulter NR, Ravn LS, et al. Liraglutide and Cardiovascular Outcomes in Type 2 Diabetes. N Engl J Med. 2016;375(4):311-22.

26. Marso SP, Bain SC, Consoli A, Eliaschewitz FG, Jódar E, Leiter LA, Lingvay I, Rosenstock J, Seufert J, Warren ML, et al. Semaglutide and Cardiovascular Outcomes in Patients with Type 2 Diabetes. N 
Engl J Med. 2016;375(19):1834-44.

27. Chaturvedi N, Stevens LK, Fuller JH, Lee ET, Lu M. Risk factors, ethnic differences and mortality associated with lower-extremity gangrene and amputation in diabetes. The WHO Multinational Study of Vascular Disease in Diabetes. Diabetologia. 2001;44(Suppl 2):65-71.

28. Lee ET, Keen H, Bennett PH, Fuller JH, Lu M. Follow-up of the WHO Multinational Study of Vascular Disease in Diabetes: general description and morbidity. Diabetologia. 2001;44(Suppl 2):3-13.

29. Nakagami T, Qiao Q, Carstensen B, Nhr-Hansen C, Hu G, Tuomilehto J, Balkau B, Borch-Johnsen K. Age, body mass index and Type 2 diabetes-associations modified by ethnicity. Diabetologia. 2003;46(8):1063-70.

30. Takeuchi M, Okamoto $\mathrm{K}$, Takagi T, Ishii $\mathrm{H}$. Ethnic difference in patients with type 2 diabetes mellitus in inter-East Asian populations: a systematic review and meta-analysis focusing on fasting serum insulin. Diabetes Res Clin Pract. 2008;81(3):370-6.

31. Spracklen CN, Horikoshi M, Kim YJ, Lin K, Bragg F, Moon S, Suzuki K, Tam CHT, Tabara Y, Kwak SH, et al. Identification of type 2 diabetes loci in 433,540 East Asian individuals. Nature. 2020;582(7811):240-5.

32. Pala L, Rotella CM. The role of DPP4 activity in cardiovascular districts: in vivo and in vitro evidence. J Diabetes Res. 2013;2013:590456.

33. Higashi Y, Gautam S, Delafontaine P, Sukhanov S. IGF-1 and cardiovascular disease. Growth Horm IGF Res. 2019;45:6-16.

34. Sudar-Milovanovic E, Zafirovic S, Jovanovic A, Trebaljevac J, Obradovic M, Cenic-Milosevic D, Isenovic ER. Hormonal Regulation of Nitric Oxide (NO) in Cardio-metabolic Diseases. Curr Pharm Des. 2017;23(10):1427-34.

35. Higashi Y, Sukhanov S, Shai SY, Danchuk S, Tang R, Snarski P, Li Z, Lobelle-Rich P, Wang M, Wang D, et al. Insulin-Like Growth Factor-1 Receptor Deficiency in Macrophages Accelerates Atherosclerosis and Induces an Unstable Plaque Phenotype in Apolipoprotein E-Deficient Mice. Circulation. 2016;133(23):2263-78.

36. Juul A, Scheike T, Davidsen M, Gyllenborg J, Jørgensen T. Low serum insulin-like growth factor I is associated with increased risk of ischemic heart disease: a population-based case-control study. Circulation. 2002;106(8):939-44.

\section{Tables}

Table 1: Baseline characteristics of study patients 


\begin{tabular}{|c|c|c|c|c|}
\hline & Overall & DPP4i (+) & DPP4i (-) & p-value \\
\hline & $n=885$ & $n=324$ & $n=561$ & \\
\hline \multicolumn{5}{|l|}{ Baseline characteristics } \\
\hline Age, years & $68.0 \pm 10.1$ & $68.1 \pm 10.0$ & $67.9 \pm 10.2$ & 0.84 \\
\hline Male, n (\%) & $733(82.8)$ & $269(83.0)$ & $464(82.7)$ & 0.90 \\
\hline Body mass index, $\mathrm{kg} / \mathrm{m}^{2}$ & $24.7 \pm 3.8$ & $24.8 \pm 4.0$ & $24.7 \pm 3.7$ & 0.50 \\
\hline Hypertension, n (\%) & $694(78.4)$ & $247(76.2)$ & $447(79.7)$ & 0.23 \\
\hline Dyslipidemia, n (\%) & $691(78.1)$ & $254(78.4)$ & $437(77.9)$ & 0.86 \\
\hline Current smoker, n (\%) & $207(23.4)$ & $72(22.2)$ & $135(24.1)$ & 0.53 \\
\hline Chronic kidney disease, n (\%) & $272(30.7)$ & $97(29.9)$ & $175(31.2)$ & 0.70 \\
\hline Hemodialysis, n (\%) & $75(8.5)$ & $23(7.1)$ & $52(9.3)$ & 0.26 \\
\hline $\begin{array}{l}\text { Acute coronary syndrome, } \mathrm{n} \\
(\%)\end{array}$ & $214(24.2)$ & $62(19.1)$ & $152(27.1)$ & 0.008 \\
\hline LVEF, \% & $60.6 \pm 12.7$ & $60.2 \pm 12.7$ & $60.7 \pm 12.7$ & 0.65 \\
\hline Diabetes duration, years & $14(6,22)$ & $13(8,20)$ & $14(5,22)$ & 0.92 \\
\hline Number of diseased vessels & $2.0 \pm 0.8$ & $1.9 \pm 0.8$ & $2.0 \pm 0.8$ & 0.12 \\
\hline \multicolumn{5}{|l|}{ Vessel location } \\
\hline RCA, n (\%) & $268(30.3)$ & $91(28.1)$ & $177(31.6)$ & 0.28 \\
\hline LAD, n (\%) & $449(50.7)$ & $176(54.3)$ & $273(48.7)$ & 0.10 \\
\hline LCX, n (\%) & $169(19.1)$ & $63(19.4)$ & $106(18.9)$ & 0.84 \\
\hline \multicolumn{5}{|l|}{ Laboratory findings } \\
\hline $\mathrm{TC}, \mathrm{mg} / \mathrm{dL}$ & $166.5 \pm 38.5$ & $160.9 \pm 33.2$ & $169.7 \pm 40.9$ & 0.001 \\
\hline LDL-C, mg/dL (Friedewald) & $96.2 \pm 31.7$ & $92.7 \pm 27.3$ & $98.2 \pm 33.8$ & 0.01 \\
\hline $\mathrm{HDL}-\mathrm{C}, \mathrm{mg} / \mathrm{dL}$ & $43.3 \pm 13.3$ & $42.6 \pm 12.5$ & $43.8 \pm 13.8$ & 0.20 \\
\hline $\mathrm{TG}, \mathrm{mg} / \mathrm{dL}$ & $119(86,163)$ & $120(85,160)$ & $119(87,163)$ & 0.63 \\
\hline $\mathrm{FBG}, \mathrm{mg} / \mathrm{dL}$ & $134.9 \pm 56.1$ & $132.9 \pm 53.1$ & $136.1 \pm 57.8$ & 0.42 \\
\hline $\mathrm{HbA} 1 \mathrm{c}-\mathrm{NG}, \%$ & $7.2 \pm 1.1$ & $7.1 \pm 1.0$ & $7.3 \pm 1.1$ & 0.02 \\
\hline hs-CRP, mg/L & $\begin{array}{l}0.09(0.03 \\
0.29)\end{array}$ & $\begin{array}{l}0.08(0.03 \\
0.30)\end{array}$ & $\begin{array}{l}0.09(0.03 \\
0.28)\end{array}$ & 0.61 \\
\hline Hemoglobin, g/dL & $13.3 \pm 1.9$ & $13.3 \pm 1.8$ & $13.3 \pm 2.0$ & 0.55 \\
\hline
\end{tabular}




\begin{tabular}{|c|c|c|c|c|}
\hline eGFR, $\mathrm{ml} / \mathrm{min} / 1.73 \mathrm{~m}^{2}$ & $69.1 \pm 28.5$ & $71.2 \pm 29.7$ & $67.9 \pm 27.8$ & 0.10 \\
\hline $1,5-A G, \mu g / m L$ & $8.6(4.1,14.4)$ & $8.7(4.1,14.7)$ & $8.6(4.1,14.3)$ & 0.69 \\
\hline \multicolumn{5}{|l|}{ Medication } \\
\hline Sulfonylurea, n (\%) & $234(26.4)$ & $99(30.6)$ & $135(24.1)$ & 0.03 \\
\hline Metformin, n (\%) & $170(19.2)$ & $77(23.8)$ & $93(16.6)$ & 0.009 \\
\hline Thiazolidinedione, n (\%) & $94(10.6)$ & $30(9.3)$ & $64(11.4)$ & 0.32 \\
\hline SGLT-2 inhibitor, n (\%) & $17(1.9)$ & $12(3.7)$ & $5(0.9)$ & 0.003 \\
\hline GLP-1 receptor agonist, n (\%) & $6(0.7)$ & $1(0.3)$ & $5(0.9)$ & 0.31 \\
\hline a-Glucosidase inhibitor, n (\%) & $190(21.5)$ & $62(19.1)$ & $128(22.8)$ & 0.20 \\
\hline Glinide, n (\%) & $72(8.1)$ & $38(11.7)$ & $34(6.1)$ & 0.003 \\
\hline Insulin, n (\%) & $223(25.2)$ & $48(14.8)$ & $175(31.2)$ & $<0.0001$ \\
\hline ACE-I/ ARB, n (\%) & $485(54.8)$ & $172(53.1)$ & $313(55.8)$ & 0.44 \\
\hline$\beta$-Blocker, n (\%) & $405(45.8)$ & $149(46.0)$ & $256(45.6)$ & 0.92 \\
\hline Statin, n (\%) & $598(67.6)$ & $242(74.7)$ & $356(63.6)$ & 0.001 \\
\hline Ezetimibe, n (\%) & $51(5.8)$ & $26(8.0)$ & $25(4.5)$ & 0.03 \\
\hline Fibrate, n (\%) & $34(3.8)$ & $11(3.4)$ & $23(4.1)$ & 0.60 \\
\hline \multicolumn{5}{|c|}{$\begin{array}{l}\text { LVEF; left ventricular ejection fraction, RCA; right coronary artery, LAD; left anterior descending artery, } \\
\text { LCX; left circumflex artery, TC; total cholesterol, LDL-C; low-density lipoprotein, HDL-C; high-density } \\
\text { lipoprotein, TG; triglycerides, FBG; fasting blood glucose, HbA1c-NG; glycated hemoglobin, hs-CRP; } \\
\text { high-sensitivity C-reactive protein, eGFR; estimated glomerular filtration rate, 1,5-AG; 1,5- } \\
\text { anhydroglucitol, SGLT-2; sodium-glucose co-transporter-2, GLP-1; glucagon-like peptide-1, ACE-l; } \\
\text { angiotensin-converting enzyme inhibitors, ARB; angiotensin receptor blockers. }\end{array}$} \\
\hline
\end{tabular}

\section{Figures}


(a)
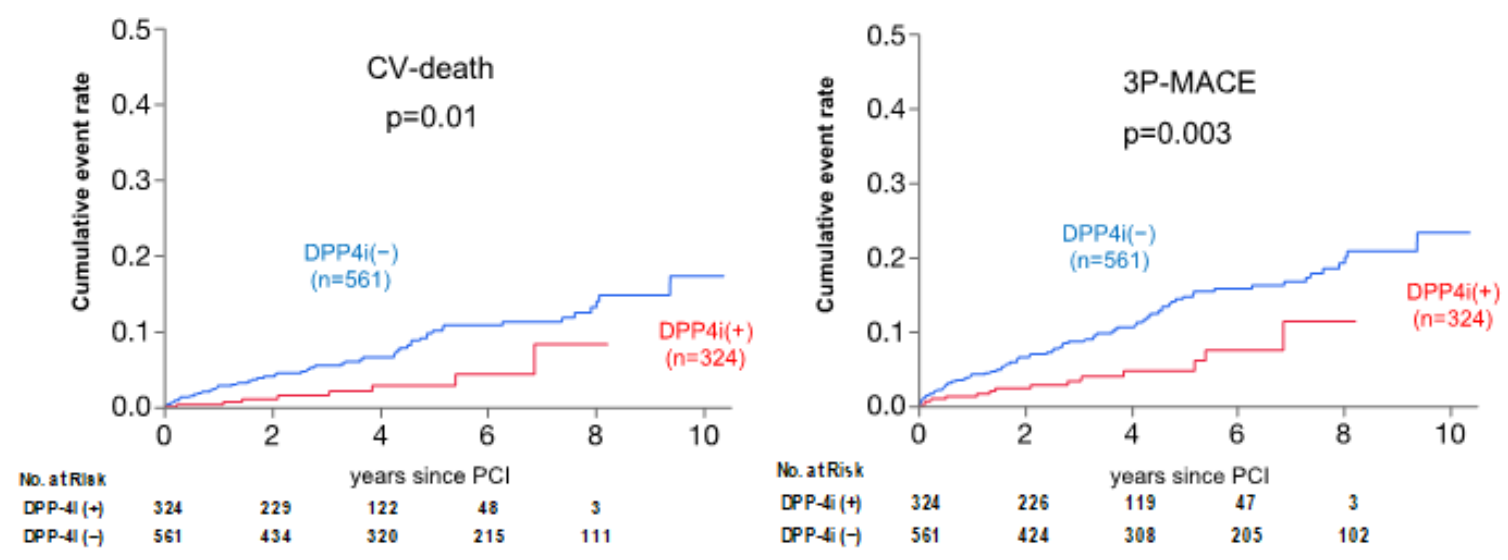

(b)
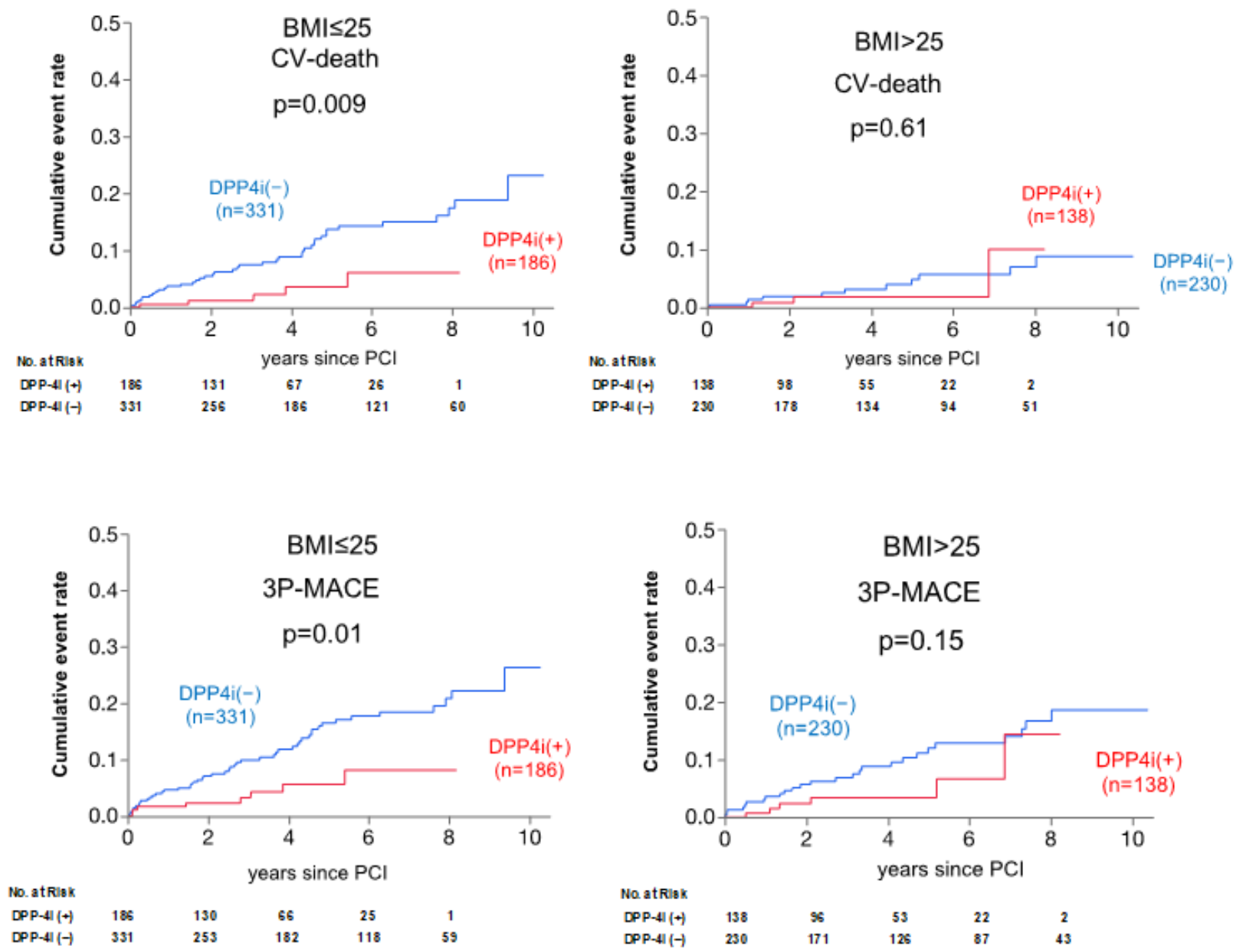

\section{Figure 1}

Cumulative incidences of adverse events following PCl in patients with and without DPP4i (a) Cumulative incidences of CV-death and 3P-MACE in patients treated with and without DPP4i (DPP4i (+) and (-) groups). (b) Cumulative incidences of CV-death and 3P-MACE in DPP4i (+) and (-) groups in patients with and without low BMI ( $\leq$ and > median BMI, 25). CV: cardiovascular, 3P-MACE: 3-point major adverse cardiovascular events, the composite of CV-death, non-fatal myocardial infarction and ischemic stroke. 


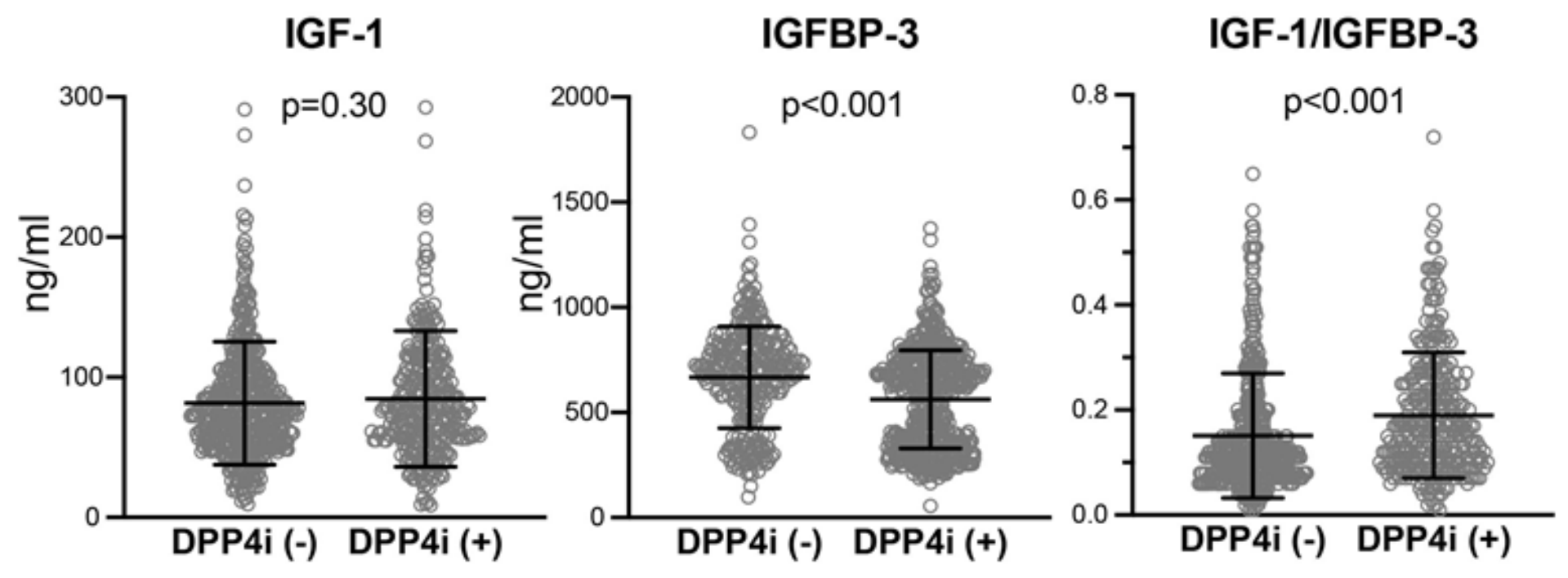

Figure 2

Comparison of serum levels of IGF-1, IGFBP-3 and IGF-1/IGFBP-3 in patients with and without DPP4i Serum levels of IGF-1, IGFBP-3 and IGF-1/IGFBP-3 ratio. Each open circle represents one participant. The horizontal lines indicate average \pm standard deviation, respectively. 
CV death

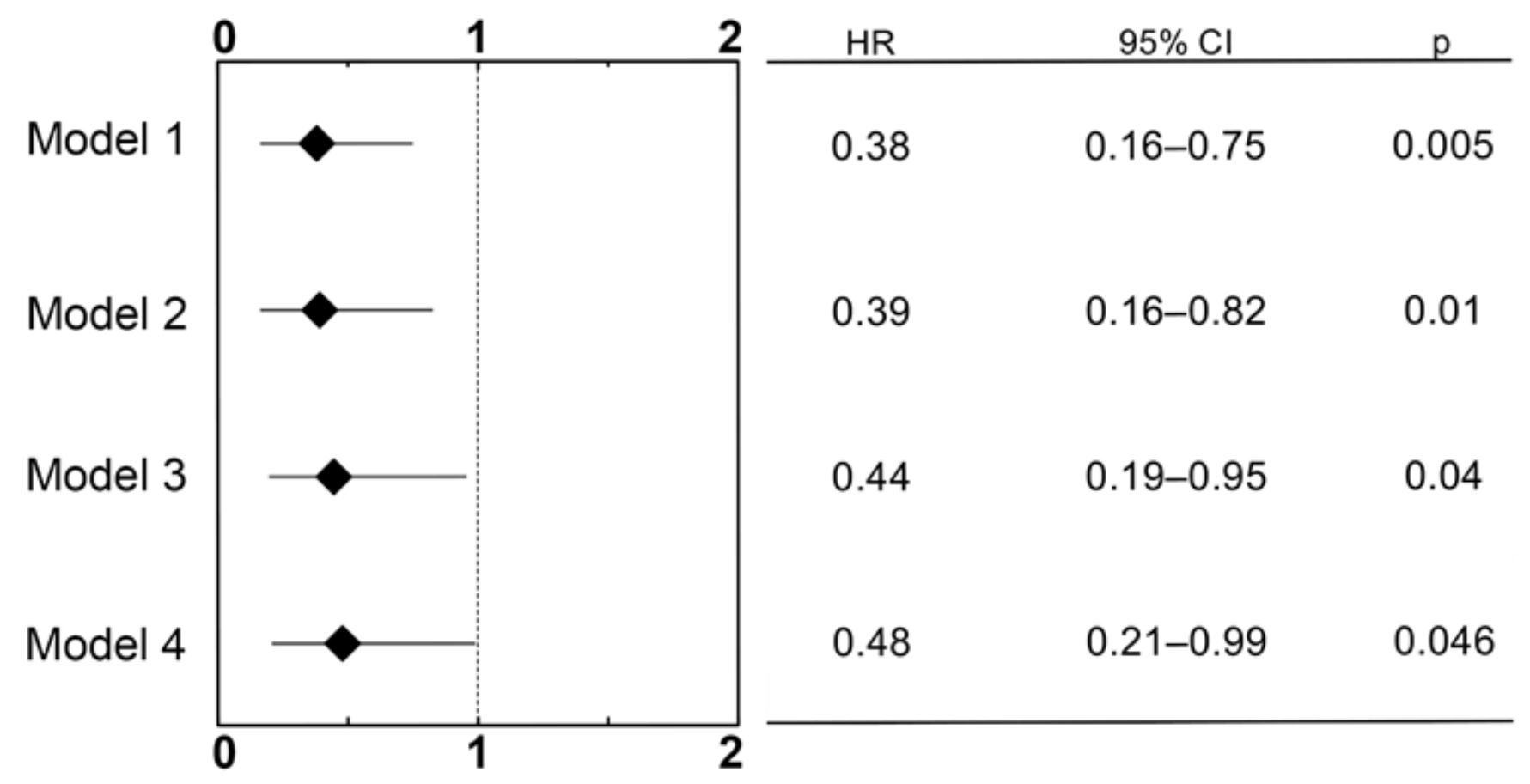

\section{P-MACE}

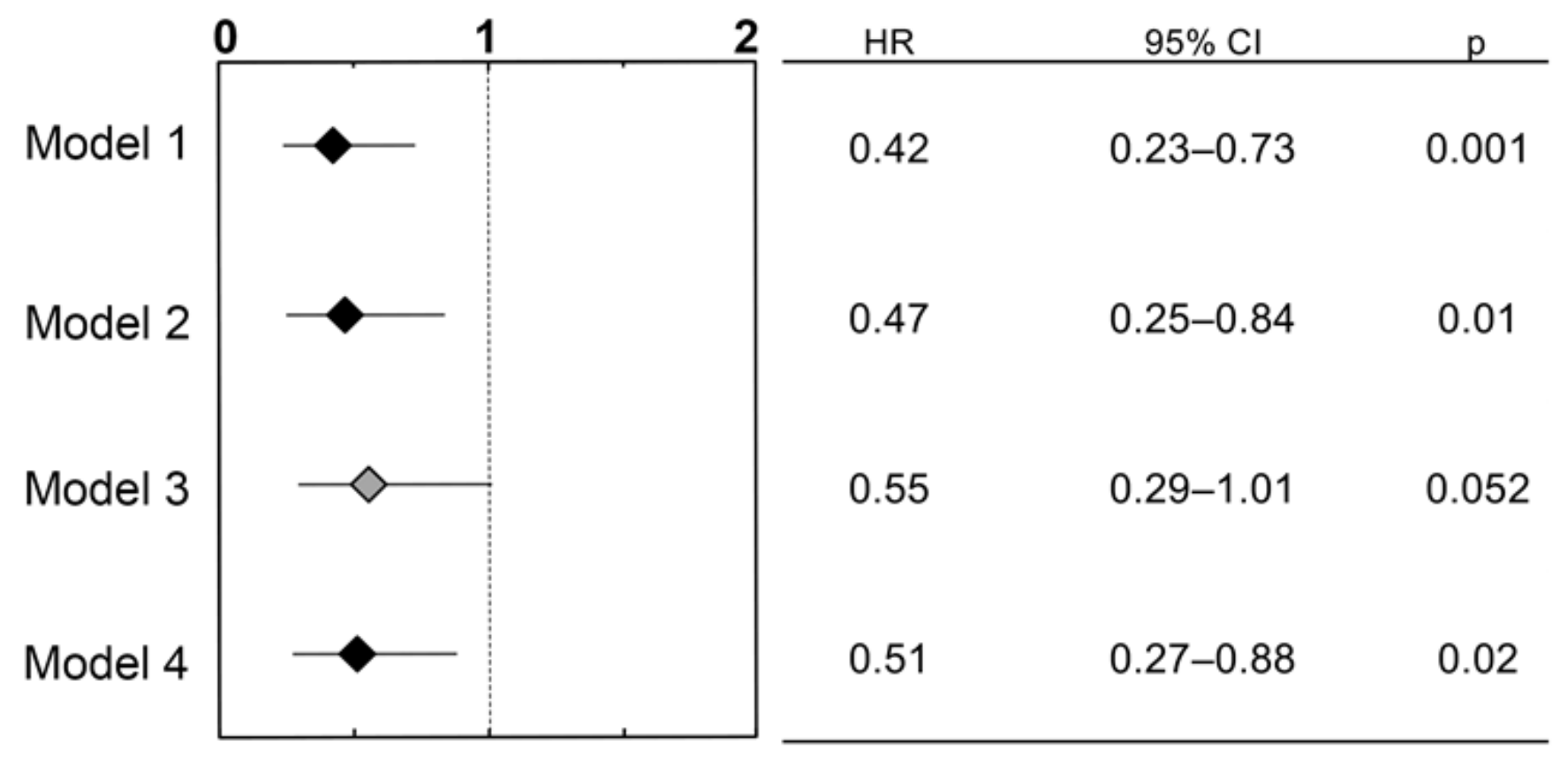

\section{Figure 3}

Prognostic impact of DPP4i at PCl for adverse CV-events in 4 Cox proportional hazard models Hazard ratios were calculated by multivariate Cox proportional hazard analyses by using 4 different models. Model 1: age, sex and DPP4i, Model 2: age, sex, body mass index (BMI), chronic kidney disease (CKD), btype natriuretic peptide (BNP), insulin and DPP4i, Model 3: age, sex, acute coronary syndrome (ACS), left ventricular ejection fraction (LVEF), statins, hemoglobin and DPP4i, Model 4: metformin, sulfonylurea, 
alpha-glucosidase inhibitors, thiazolidinedione, glinide, insulin and DPP4i. HR: hazard ratio, $95 \% \mathrm{Cl}$; confidence interval.

\section{Supplementary Files}

This is a list of supplementary files associated with this preprint. Click to download.

- SENDOK20210610DPP4iXIGF1SupplementarymaterialsCVDB.docx 